\title{
Vorwort und Benutzungshinweise
}

Die „Entscheidungen in Kirchensachen seit 1946“ (KirchE) wurden im Jahre 1963 von Carl Joseph Hering, damals Ordinarius für Kirchenrecht an der Rechtswissenschaftlichen Fakultät der Universität zu Köln, und seinem Assistenten Hubert Lentz gegründet.

Die Sammlung veröffentlicht Judikatur deutscher staatlicher Gerichte aus allen Zweigen der Rechtsprechung zum Verhältnis von Kirche und Staat und zu weiteren Problemkreisen, die durch die Relevanz religiöser Belange gekennzeichnet sind. Angesichts dieses breiten Themenkatalogs kann eine Vollständigkeit dieser Sammlung nur angestrebt werden, wenn man eine gewisse zeitliche Distanz in Kauf nimmt.

Die steigende Bedeutung religionsrechtlich relevanter Fragen in der Rechtsprechung des Europäischen Gerichtshofs für Menschenrechte (EGMR) und des Europäischen Gerichtshofs (EuGH) macht es unerlässlich, auch deren Judikatur zu berücksichtigen. Dabei werden vornehmlich solche Entscheidungen ausgewählt, die aus Verfahren in Deutschland hervorgegangen oder sonst von grundsätzlicher Bedeutung sind. Die Veröffentlichung erfolgt in einer Amtssprache oder amtlichen Übersetzung, die der Gerichtshof für die amtliche Ausgabe der jeweiligen Entscheidung verwendet hat. Ebenso bleibt die von der deutschen Praxis abweichende Form der Entscheidungen und der Abkürzungen gewahrt. Die in der Zeit von 1965 bis 2001 ergangenen Entscheidungen des EGMR und EuGH zu religionsrechtlichen Fragen sind in einem Sonderband 42 KirchE-EU (2007) dokumentiert.

In Fußnote 1 finden sich Angaben zu den Leitsätzen und Quellen in amtlichen Entscheidungssammlungen und Fachzeitschriften, ggf. auch Hinweise auf Parallelverfahren. Eine digitale Zugriffsmöglichkeit wird für BeckRS, HUDOC, juris, RWR und, soweit eine andere Veröffentlichung nicht ermittelt werden konnte, für die Justizdatenbank des betreffenden Landes nachgewiesen. Soweit ein Verfahren mit der hier abgedruckten Entscheidung nicht zum Abschluss gelangt ist, erscheint in Fußnote 1 ein Hinweis auf den weiteren Verlauf. Etwaige weitere Entscheidungen aus dem Rechtsmittelzug etc., soweit sie wesentliche Ausführungen zu religionsrechtlichen Fragen enthalten, entnehme man späteren Bänden von KirchE. Kurzgefasste Rechtsmittelentscheidungen, die sich im Wesentlichen auf ergänzende Ausführungen beschränken, werden bei der Vorinstanz in Fußnote 1 referiert.

Entscheidungen zum Sonn- und Feiertagsrecht, in denen religiöse Aspekte relevant sind, werden aus Raumgründen in der Regel nur mit einem Urteil etc. im Volltext dokumentiert; weitere einschlägige Entscheidungen im Veröffentlichungszeitraum sind dort in der Fußnote 1 nachgewiesen. Dasselbe gilt für Entscheidungen aus dem Arbeitsrecht, die - wie beispielsweise auf dem Gebiet der Eingruppierung von Dienstkräften - nur am Rande kirchentypische Merkmale aufweisen. Entscheidungen zum Asylrecht werden nur berücksichtigt, soweit sie religionsrechtliche Verhältnisse in Deutschland oder der Europäischen Union betreffen, etwa den Nachweis des Übertritts zu einer christlichen Konfession als Nachfluchtgrund. Für Erkenntnisse über die religionspolitische Lage in den Herkunftsländern, die in der Judikatur vielfach erörtert werden, stehen der Praxis anderweitige Zugänge offen. 
Die Herausgeber haben die Sammlung als Judikatur-Archiv konzipiert. Für die Aufnahme einer Entscheidung ist maßgebend, ob der Verfahrensgegenstand und die religionsrechtlichen Erwägungen für Wissenschaft und Praxis von Interesse sind. Deshalb wurden zum Teil auch instanzgerichtliche Entscheidungen berücksichtigt, die im weiteren Verlauf des Verfahrens keinen Bestand hatten.

Soweit die als amtlich gekennzeichneten Leitsätze der Gerichte verwendet wurden, ist dies vermerkt. Im Übrigen wurden die Leitsätze möglichst am religionsrechtlich relevanten Inhalt der Entscheidung orientiert. Dasselbe gilt für die Fassung des Sachverhalts und der Prozessgeschichte und eine eventuelle Kürzung der Entscheidungsgründe. Der z.T. unterschiedliche Zitier- und Abkürzungsmodus ist nur angeglichen, wo Verwechslungen in Betracht kommen. Eine in der amtlichen Fassung oder bei juris benutzte Randbezifferung ist in den Entscheidungsgründen (kursiv und in eckigen Klammern) berücksichtigt. Das Abkürzungsverzeichnis wurde im Wesentlichen auf Zeitschriften beschränkt. Zur Auflösung von weiteren juristischen Abkürzungen wird auf Kirchner, Abkürzungsverzeichnis der Rechtssprache, 7. Aufl., Berlin 2012, verwiesen. Soweit in den Urteilen etc. auf andere Entscheidungen, die auch in KirchE abgedruckt sind, Bezug genommen wird, ist die Fundstelle durch einen entsprechenden Zusatz nachgewiesen. Über die in den Bänden 1-25 erschienene Judikatur informiert zusätzlich ein im Jahr 1993 erschienener Registerband.

Zugänge zur Judikatur kirchlicher Gerichte, die in dieser Sammlung schon aus Raumgründen nicht berücksichtigt werden kann, eröffnen die Rechtsprechungsbeilage zum Amtsblatt der EKD (bis 2013 jeweils Beilage zu Heft 4 eines Jahrganges), danach das elektronische Fachinformationssystem FIS zum Kirchenrecht (unter www.kirchenrechtekd.de Rubrik „Rechtsprechung“), und die kirchenrechtlichen Fachzeitschriften, insbesondere das „Archiv für katholisches Kirchenrecht“, „Kirche und Recht“ und die „Zeitschrift für evangelisches Kirchenrecht“. Im Bereich der Katholischen Kirche ist die Spruchpraxis arbeitsrechtlicher Schiedsstellen und Arbeitsgerichte, d.h. der Kirchlichen Arbeitsgerichte Erster Instanz, des Kirchlichen Arbeitsgerichtshofs und des Delegationsgerichts der Apostolischen Signatur (Tribunal Delegatum et a Supremo Signaturae Apostolicae Tribunali Constitutum), u.a. aus der Zeitschrift „Die Mitarbeitervertretung“ und der „Zeitschrift für Arbeitsrecht und Tarifpolitik in Kirche und Caritas“ sowie über die Homepage der Deutschen Bischofskonferenz zu erschließen.

Seit seiner Gründung erscheint das Werk in Zusammenarbeit mit dem Institut für Kirchenrecht und rheinische Kirchenrechtsgeschichte der Universität zu Köln und wird dort auch redaktionell betreut. Den Mitarbeiterinnen und Mitarbeitern schulden die Herausgeber für ihren Einsatz herzlichen Dank.

Den Benutzern der Sammlung sei versichert, dass die Herausgeber kritische Anmerkungen und Hinweise auf unveröffentlichte Entscheidungen sehr zu schätzen wissen.

Köln, im Herbst 2018

Stefan Muckel

Manfred Baldus 